\title{
Biomechanical Role of Bone Anisotropy Estimated on Clinical CT Scans by Image Registration
}

Elham Taghizadeh¹; Mauricio Reyes ${ }^{1}$; Philippe Zysset ${ }^{1}$; Adeliya Latypova ${ }^{2}$; Alexandre Terrier $^{2}$ and Philippe Büchler ${ }^{1}$

${ }^{1}$ Institute for Surgical Technology \& Biomechanics, University of Bern, Switzerland.

${ }^{2}$ Laboratory of Biomechanical Orthopedics, École Polytechnique Fédérale de Lausanne, Lausanne, Switzerland

The authors have no commercial, proprietary, or financial interest in any products or companies described in this article.

Address for correspondence:

Philippe Büchler

Institute for Surgical Technology \& Biomechanics

University of Bern

Stauffacherstrasse 78

3014 Bern, Switzerland

Email: philippe.buechler@istb.unibe.ch 


\section{ABSTRACT}

Image-based modeling is a popular approach to perform patient-specific biomechanical simulations. Accurate modeling is critical for orthopedic application to evaluate implant design and surgical planning. It has been shown that bone strength can be estimated from the Bone Mineral Density (BMD) and trabecular bone architecture. However, these findings cannot directly be fully transferred to patient-specific modeling since only BMD can be derived from clinical CT. Therefore, the objective of this study was to propose a method to predict the trabecular bone structure using a $\mu \mathrm{CT}$ atlas and an image registration technique. The approach has been evaluated on femurs and patellae under physiological loading. The displacement and ultimate force for femurs loaded in stance position were predicted with an error of $2.5 \%$ and $3.7 \%$ respectively, while predictions obtained with an isotropic material resulted in errors of $7.3 \%$ and $6.9 \%$. Similar results were obtained for the patella, where the strain predicted using the registration approach resulted in an improved mean squared error compared to the isotropic model. We conclude that the registration of anisotropic information from of a single template bone enables more accurate patient-specific simulation from clinical image datasets than isotropic model.

Keywords: Trabecular bone, Femur, Patella, Anisotropy, Finite element analysis (FEA) 


\section{Introduction}

Finite element models are important to estimate bone strength, predict fracture risk, and improve orthopedic devices. Several studies have proposed simulation tools to plan or to predict the outcome of orthopedic surgeries ${ }^{4,5,22,31}$. Mechanical models of the bone rely on bone mineral density (BMD) obtained on CT scan images. However, it has been shown that BMD alone is not enough to accurately predict trabecular bone strength and including trabecular anisotropy to the FE simulations improves the accuracy of bone simulations over isotropic models ${ }^{25,27}$. Maquer and colleagues calculated morphology and elastic constants of more than 700 cubic samples of trabecular bone using $\mu \mathrm{FE}$ simulations and showed that bone volume fraction (BV/TV) and anisotropy determine the elastic properties with $R^{2}=0.968^{21}$. The bone volume fraction (BV/TV) and fabric anisotropy describe $98 \%$ of the variations in trabecular bone elastic properties ${ }^{21}$. BVITV alone describes about $90 \%$, while the anisotropic architecture accounts for about $8 \%$ of the bone elastic properties. The trabecular bone structure is adequately characterized in high-resolution $\mu \mathrm{CT}$ images ${ }^{2,3}$, which require high dose of radiation and limit their use to cadaveric specimens or biopsies from patients. In addition, the trabecular bone structure can be estimated in vivo in the peripheral skeleton, such as the distal radius or tibia with high-resolution peripheral quantitative tomography (HR-pQCT) ${ }^{23}$. Alternative techniques are required to include anisotropic information to predict femoral and vertebral fracture risks, which are related to osteoporosis or to evaluate the outcomes of the frequent hip and knee replacements. 
Different methods have been proposed to extract bone architecture from clinical level CT images. Gradient Structure Tensor (GST) ${ }^{30}$ or Sobel Structure Tensor (SST) ${ }^{14}$ were used to calculate the orientation and the degree of anisotropy of trabecular bone $e^{6,14,17,34}$. Larsson et al. ${ }^{17}$ studied the correlation between GST at the clinical level and bone anisotropy calculated from $\mu$ CT scans. They showed that the GST method calculates the bone anisotropy accurately when the Degree of Transverse Isotropy (DTI) is larger than a specific threshold. However, the optimum value of this threshold is large and the anisotropy can only be quantified on the few bone regions having a strong bone anisotropy. These methods were also developed using imaging data that were not acquired clinically on patients, and since their accuracy depends on the image resolution, changes to the image acquisition protocol can affect the results.

Instead of trying to extract bone anisotropy directly form the clinical images, alternative approaches have been proposed to obtain it from descriptors of bone surface ${ }^{16}$ or from X-ray attenuation coefficient ${ }^{10}$. Such an approach has been used to determine bone anisotropy of the human mandibular, but accuracy of the predicted anisotropy was not reported. Other authors suggested using iterative FE analyses or micromechanics to predict the anisotropic information from the principal directions of the calculated stress tensor $^{9,27,33}$. Another set of studies suggests to predict this information alternative approaches have been proposed to obtain bone anisotropy from a database of cadaveric samples ${ }^{8,19}$. Hazrati et al. $^{8}$ chose the closest BV/TV distribution to the patient's bone in a bone database and morphed the anisotropic information from this cadaveric reference to the patient's bone. A regression method was proposed by Lekadir et al. ${ }^{19}$ where the structural information of trabecular bone is predicted through 
features derived from bone volume fraction and local shape deformations. Although both methods produce good estimates of bone anisotropy, a large database of highresolution scans is needed to cover the variability of shape and intensities observed in the population. In addition, both methods rely on the BV/TV extracted from HRpQCT images to estimate bone architecture, which is not applicable in vivo.

Therefore, the objective of the present study was to estimate bone anisotropy based on a patient CT scan and the high-resolution scan of a single template of bone anisotropy. Our hypothesis was that the gross anisotropic information relevant to determine bone strength could be mapped from one template reference to patients' data using image registration, which alleviates the need of a large bone database. In this work, the accuracy of finite element simulations based on a template bone anisotropy was evaluated in situations involving different anatomical locations and CT image quality.

\section{Materials and Methods}

The anisotropic information of the trabecular bone was estimated based on one highresolution image, $\mu \mathrm{CT}$ or HRpQCT, which was used as template bone. The image of this template bone was registered to the patient's clinical CT image. After this step, the registered image had the same shape as the patient's bone, while the structure was transformed from the high-resolution template. Finally, the anisotropic information was extracted from the registered image. The accuracy of the method was evaluated by comparing its mechanical outcomes with calculations directly obtained from highresolution scan of the target bone. Tests were conducted on cubic trabecular samples cropped from the center of femoral heads and on the proximal femur and on patellae. 


\section{Datasets}

\section{Femur database}

A database of 11 left proximal femurs were obtained from Luisier et al. ${ }^{20}$. The proximal femurs were collected from six male and five female donors. The donors' ages were in the range of $[63,93]$ years with the average age of $78.7 \pm 10.8$ years. From each bone one HRpQCT (Xtreme-CT, Scanco, Switzerland) image with the spatial resolution of $0.082 \times 0.082 \times 0.082 \mathrm{~mm}$ and QCT (Brilliance64, Philips, Germany) image with spatial resolution of $0.32 \times 0.32 \times 1 \mathrm{~mm}$ were acquired. The high-resolution images were scanned with an intensity of $900 \mu \mathrm{A}$ and voltage of $60 \mathrm{kV}$. The BMD was calculated based on the intensity $(\mathrm{HU})$ values obtained using the manufacturer's calibration function and provided in the image header ${ }^{20}$. The QCT scans were performed with an intensity of 100 $\mathrm{mA}$ and a voltage of $120 \mathrm{kV}$. A calibration phantom (BDC phantom, QMR GMbh, Germany) was also scanned with the bones to convert the image intensity to BMD values.

The database covers large bone shape variability. The head diameter $(d)$ and neck angle $(\theta)$ were measured to describe the femurs shape; $d \in[40.02,51.33] \mathrm{mm}$ with the average and standard deviation of $45.6 \pm 3.9 \mathrm{~mm}$ and $\theta \in[114.10,135.70] \mathrm{mm}$ with average and standard deviation of $126.8 \pm 6.0$ degrees. One bone without any pathology was arbitrarily chosen from the database as template. The resolution of the QCT was decreased to represent a standard clinical CT acquisition; the QCT scans were resampled from $0.32 \times 0.32 \times 1.0 \mathrm{~mm}^{3}$ to $1.25 \times 1.25 \times 1.0 \mathrm{~mm}^{3}$. 


\section{Patella database}

The patella database contains 11 patellae from five female and six male donors with age in the range of $[54,93]$ years. Average and standard deviation were $71.0 \pm 11.6$ years. Each patella was wrapped in saline soaked gauze and QCT images with the clinical resolution of $0.39 \times 0.39 \times 0.625 \mathrm{~mm}^{3}$ were acquired with a calibration phantom (BDC phantom, QMR Gmbh, Germany). For each bone, a $\mu \mathrm{CT}$ scan was acquired (Skyscan 1076, Bruker $\mu \mathrm{CT}$, Kontich, Belgium) with a spatial resolution of $18.3 \mu \mathrm{m}$. The scanning settings were $80 \mathrm{kV} / 120 \mathrm{~mA}, 540 \mathrm{~ms}$ exposure time, $1 \mathrm{~mm}$ aluminum filter, and 0.2 degrees rotation step. For memory management, the $\mu \mathrm{CT}$ scans were downsampled from $0.018 \times 0.018 \times 0.018 \mathrm{~mm}^{3}$ to $0.037 \times 0.037 \times 0.037 \mathrm{~mm}^{3}$ spatial resolutions. One healthy bone was chosen as template for image registration.

In both datasets the high-resolution scans (HRpQCT for proximal femurs and $\mu C T$ scans from patellae) of the test bones were used as ground-truth to validate the results.

\section{Image Registration}

The aim of the image registration step is to translate, scale and deform the template image such that it overlaps the target image from the patient. The non-rigid registration is used to find the corresponding points between two samples. For non-rigid registration, image intensity information is used to derive the metric for evaluating the quality of the registration at each iteration. This intensity information includes edges, or gradient information, as well as density information present in the entire volume. For Image registration we used normalized correlation as metric, which considers a statistical relation between image intensities ${ }^{15}$. 
Once image registration is performed, the corresponding voxels in both images occupy the same spatial volume. In our problem, the template and target bone images are from different patients and have been captured using different devices (QCT/CT and HRpQCT/ $\mu C T$ scanner). Subsequent rigid, affine and non-rigid registrations have been applied to the patients' images (Figure 1).

\section{Image registration for the proximal femur}

Due to the size of the HRpQCT images, non-rigid image registering is computationally demanding (or even infeasible). Therefore an intermediate step has been added to the registration procedure. Instead of $\mathrm{HRpQCT}$, the lower resolution QCT image of the template bone was registered to the patient's calibrated CT scan. The non-rigid transformation, calculated from the template QCT registration is then applied to the template HRpQCT image. Since both QCT and HRpQCT images were acquired from the same bone, this transformation results in the same bone shape, but with higher resolution.

Rigid and affine registrations were applied to the template image to initialize the nonrigid registration. Four landmarks positioned on the images were used to calculate the rigid alignment of the images ${ }^{12}$. The landmarks were positioned on the femur head fovea, the most upper part of the greater trochanter, the lesser trochanter and in the center of the shaft below the lesser trochanter. The New-Block Matching method ${ }^{24}$ was used for the affine registration. For non-rigid registration, the B-spline image registration ${ }^{26}$ implemented in Elastix ${ }^{15}$ was used. This method uses grid-based calculations to achieve computational efficiency. After non-rigid registration of the QCT template image to the patient's CT image, the deformation fields from affine and B- 
spline transformations were applied to the HRpQCT images. This process results in an artificial high-resolution HRpQCT morphed into the shape of the patient's femur.

\section{Image registration for the complete patella}

The registration of the patella followed a similar pipeline as described for the femur. The only difference is that only $\mu \mathrm{CT}$ and patient's clinical CT scans were available for the patella dataset. For computational efficiency, the $\mu \mathrm{CT}$ scans were down-sampled from $0.037 \mathrm{~mm}$ to $0.074 \mathrm{~mm}$ isotropic spacing. The cortical and trabecular bone were manually segmented and the image registration was only performed on the trabecular bone. Similar to the femur registration, after rigid and affine registration for initial alignment, the B-spline based non-rigid registration was used to register the downsampled template $\mu \mathrm{CT}$ scan of the trabecular bone to the CT scan of the patient. In the final step, the transformation was applied to the template $\mu \mathrm{CT}$ scan.

To quantify the error on predicting trabecular bone structure, we calculated the Frobenius norm of the error tensor as follows:

Error $_{\text {Tensor }}=\frac{\left\|\mathbf{M}-\mathbf{M}^{\prime}\right\|}{\|\mathbf{M}\|}$

where $\mathbf{M}$ is the ground-truth fabric tensor and $\mathbf{M}^{\prime}$ corresponds to the predicted bone fabric tensor.

\section{Mechanical simulations}

Finite element simulations were used to evaluate the effect of the anisotropic predictions on bone mechanics. Several test cases were considered and for each one, 
the mechanical predictions were compared to the mechanical behavior calculated using the homogenized models created from the original high-resolution scan of the patient's bone (ground-truth).

\section{Cube study}

The accuracy of the predicted anisotropy was evaluated on cubic samples under various loading conditions. Cubic samples having an edge length of 260 voxels (21.3 $\mathrm{mm}$ ) were selected from the center of each femoral head. This size corresponds to the largest cube that could fit in the smallest femoral head while leaving some margins to the head border. The cubes were cut in the same position in both the original HRpQCT and on the template HRpQCT registered to the patient's bone. A mesh with 512 threedimensional hexahedral elements (C3D8R) was used for the numerical model. The material properties of each bone were obtained from its corresponding image, and assigned to each finite element. The bone anisotropy was obtained using a $2.5 \mathrm{~mm}$ grid overlapped on the image. The material anisotropic tensor was calculated with the GST method $^{30}$ on $5.3 \mathrm{~mm}$ spheres centered on each grid node. For each element, the fabric tensor was calculated by interpolating the fabric tensors from the neighbor grid nodes to the center of the element. Zysset's elastic material model ${ }^{35}$ (equation (2)) was used to define the material properties of each element.

$$
\begin{aligned}
\epsilon_{i} & =\epsilon_{0} \rho^{k} m_{i}^{2 l}, \\
\nu_{i j} & =\nu_{0} \frac{m_{j}^{l}}{m_{i}^{l}}, \\
\mu_{i j} & =\mu_{0} \rho^{\kappa} m_{i}^{l} m_{j}^{l}
\end{aligned}
$$


where, $\epsilon_{i}, v_{i j}$ and $\mu_{i j}$ are the orthotropic engineering constants, $\epsilon_{0}=16.56 G P a, v_{0}=$ 0.18, $\mu_{0}=5.59 \mathrm{GPa}, l=1.72$ and $\kappa=1.84$ are the model parameters, $m_{i}$ are the eigenvalues of the bone's fabric tensor and $\rho$ is the bone volume fraction (BV/TV). The predicted anisotropy described as the fabric tensor was quantified on the registered image and the BV/TV value was extracted from the patient's bone scan. After assigning material properties, each cube was loaded in six canonical modes (three orthogonal compression and three orthogonal shear) with $5 \%$ strain. The von Mises stress value then was calculated for each element to compare the accuracy of different methods (Figure 2).

\section{Proximal femur study}

The effect of the predicted bone fabric was also evaluated on the overall behavior of the proximal femur loaded in stance position. In this part, the finite element model proposed and validated by Luisier et al. ${ }^{20}$ was used. Prediction of the mechanical simulations output for the failure of the full bone, using the trabecular bone anisotropy estimated with our method, was analyzed and compared with the mechanical behavior obtained with the bone architecture extracted from the actual HRpQCT images and with an isotropic model (Figure 3).

Each bone was meshed with the automatic bone meshing method introduced by Pahr and Zysset ${ }^{25}$. First, a very coarse and smooth surface mesh was created. Then this surface mesh and cortex labels were used as input to the bone mesher. The surface mesh was extruded in the direction normal to the surface on a distance corresponding to the cortical layer. Since the trabecular bone was meshed with tetrahedral elements, 
extruding the triangular surface mesh results in a cortical mesh based on triangular prisms. The thickness of cortical layer was defined by the cortical bone segmentation.

The image intensity values must be calibrated to compute the BV/TV from the Bone Mineral Density (BMD (mg/cc)) extracted from the HRpQCT image. The following calibration function was used to relate the normalized BMD (nBMD) to the corresponding BV/TV from HRpQCT ${ }^{20}$. The $\mathrm{nBMD}$ was calculated by dividing the BMD by its maximal value of $1400 \mathrm{mg} / \mathrm{cc}$.

$$
\begin{aligned}
B V / T V_{\mu C T} \cong & -6.100 \times n B M D^{4} \\
& +5.573 \times n B M D^{3} \\
& -0.930 \times n B M D^{2} \\
& +1.420 \times n B M D
\end{aligned}
$$

The same approach used for grid interpolation in the cube study has been used to assign the bone fabric for each element of the mesh. The anisotropic information was extracted using the MIL method ${ }^{7}$. The bone material model introduced by Schwiedrzik and Zysset $^{28}$ was used to compute the non-linear bone material properties based on the local anisotropic information and BV/TV values (Table 1).

The femurs were loaded in the stance position. The lower part of proximal femur was fixed to avoid bone shifting and a $2.5 \mathrm{~mm}$ displacement was applied on top of the head with a velocity of $1 \mathrm{~mm} / \mathrm{sec}$. The reaction force as well as the bone stress and failure were computed and used to evaluate the accuracy of the anisotropic predictions.

\section{Patella study}

Each patella was meshed with tetrahedral element having an average element size of about $2 \mathrm{~mm}$. Meshing was performed using the CGAL mesh creator $^{32}$. A sensitivity 
analysis was done and showed that an element size of $2 \mathrm{~mm}$ is sufficient for convergence of the FE solution. Similar to the proximal femur study, the MIL method was used to calculate the material orientation for each element of the mesh.

In our study elements with high bone volume fraction $(\mathrm{BV} / \mathrm{TV}>0.6)$ were assumed as cortical bone and assigned isotropic material to these elements. For the rest of the elements, we used the predicted anisotropic information obtained using the proposed image registration method. The anisotropic information was assigned to each element using the same method as described for the femur case. For the patella, the grid distance and sphere size were $2.5 \mathrm{~mm}$ and $5.0 \mathrm{~mm}$, respectively. The same material model and mechanical parameters as for the femur study were used for the patella (Table 1).

The boundary condition corresponded to loading of the non-resurfaced patella in total knee replacement (TKA) during squat at 60 degrees knee flexion ${ }^{18}$. The model included the patella with cartilage, femoral surface, four quadriceps muscles and patellar tendon. The cartilage was created by 3-mm extrusion of posterior articular surface of the bone. The muscles and tendon were modeled by four and two connector elements, respectively. The femur, muscle origins, and tibia tendon attachment were fixed, while the patella had all degrees of freedom and was constrained by the contact with the femoral surface. The muscle forces were applied on the corresponding connectors (Figure 4). The quadriceps and tendon forces were then distributed along the patellar anterior surface through corresponding attachment points with cubic weighting method. Logarithmic maximum principal strain and von Mises stress were computed for both the 
predicted and true anisotropic model extracted from the original $\mu \mathrm{CT}$ scan from the patient and compared element-wise to validate the predictions.

\section{Results}

\section{Image Registration}

The quality of the image registration was first estimated by comparing the microstructure of the registered image with its corresponding high-resolution image (groundtruth). The predicted directions for the trabecular bone structure looked very similar to the target image (Figure 5). When comparing the predicted images with the highresolution ground-truth, the major difference concerns the bone volume fraction. This difference comes from the fact that the bone volume fraction of the selected reference dataset differs from the target images. However, this aspect does not affect the predictions of the proposed technique as only the bone architecture is extracted from the template, while the bone density is extracted from the patients' images.

To provide a quantitative estimation of the error introduced by the registration procedure, the orientation and fabric tensor were computed for our method and compared against the ground-truth. Our method predicted the fiber directions for 10 femoral trabecular bones with $29.7 \pm 3.3$ degrees and norm of the tensor with $14.1 \% \pm$ $4.0 \%$ in average. For the 10 patellar bones the principal direction of the trabecular bone was predicted with an average error of $24.4 \pm 3.0$ degrees. The fabric tensors were predicted with the error equal to $14.8 \% \pm 1.5 \%$. In addition, the average error on the predicted bone directions was lower for elements having high degree of anisotropy (DA) 
values. This means that the regions of strong bone anisotropy are predicted more accurately than the trabecular regions exhibiting bone architecture close to an isotropic distribution.

\section{Mechanical Simulation}

\section{Cube study}

Six mechanical loadings were applied to the cubes extracted from the center of the femoral head for 1) material properties extracted from the original HRpQCT image, 2) material properties predicted using our method and 3) with the material assumed isotropic. Our method predicted the von Mises stress value in average with $9 \%$ error, where the average error with the isotropic material was 14\% (Table 2). In addition, the error using the isotropic material increased to $19.7 \%$ when the loading direction was close to the direction of the trabecular bone, while in most of the cases the accuracy of our method was not affected by the direction of loading (Table 2). Overall the proposed method improved the accuracy of Mises stress prediction compared to isotropic material by about $10 \%$ when the loading is in the direction of the fibers. The pairwise t-test showed a significant difference between the accuracy of the proposed method and the isotropic material assumption $(p<0.001)$.

\section{Proximal femur}

To better analyze the accuracy of the method on the overall mechanical predictions in a realistic loading scenario, the error on force and displacement at the point of failure (the maximum force in the force-displacement curve) was calculated for human femur loaded in a stance configuration. Including the predicted anisotropy improved the failure 
prediction compared to the isotropic material (Figure 6). In average, the proposed method predicted the displacement at failure with an error of $2.5 \%$, while an isotropic model predicted the displacement with an average error of $7.3 \%$. The proposed method also improved the failure force from $6.9 \%$, obtained with an isotropic model, to $3.7 \%$. The correlation of the predicted ultimate force and the ground-truth was also improved using the template registration compared to the isotropic material. The correlation with the ground-truth was 0.99 for both isotropic and our method. However, while the slope of the correlation was 0.99 for the template registration, the ultimate force was consistently underestimated by the isotropic material model (Figure 7). The pairwise ttest showed a significant difference between the ultimate forces calculated with the isotropic material and our method $(p$-value $=0.001)$. There was also a significant difference between the maximal force calculated with the isotropic model and the ground-truth $(p$-value $=0.001)$, while no significant difference could be shown between our method and the ground-truth $(\mathrm{p}$-value $=0.249)$.

\section{Patella}

The maximal principal logarithmic strain was used to evaluate the accuracy of our method in predicting anisotropic information. The strain values for a typical bone are shown in Figure 8. Results showed that the template registration method has a higher correlation with the ground-truth in comparison with using isotropic material. Our method also improved the slope of the linear regression to 1.02 compared to isotropic model with the slope of 1.13 .

On average isotropic material properties predicted the principle component of logarithmic strain values at each node for 10 test patellae with an error of $13.2 \% \pm 6.0 \%$ 
and normalized mean squared error (NMSE $=(x-y)^{2} / \sigma_{x}^{2}$, where $x$ is the ground-truth, $y$ the predicted value and $\sigma_{x}^{2}$ represents the variance of the ground-truth) of $21 \%$. The proposed registration method improved the prediction of bone strain to $9.7 \% \pm 2.2 \%$ error with NMSE of $10 \%$. The error on the von Mises stress predictions were $8.6 \% \pm$ $2.7 \%$ for the isotropic material and $6.7 \% \pm 1.3 \%$ for the template registration. Pairwise $t-$ test showed a significant difference between accuracy of our method and isotropic material properties $(p$-value $=0.045)$

\section{Discussion}

In this study, we proposed to use image registration for predicting anisotropic information of a patient's CT scan. This method predicted the trabecular bone's main direction with an average error of 27 degrees and the tensors were predicted with $14 \%$ error. Although the error on the prediction of the trabecular orientation appears to be important, the values obtained with a single template were in the same range as values reported in the studies of Hazrati and colleagues $^{8,19}$, who relied on a large bone database.

We observed that the error on predicted anisotropy is higher in regions of the bone with very low bone mineral density. However, the high prediction error in these regions does not influence the overall biomechanical behavior of the bone. To analyze how the error made when predicting the bone orientation influences biomechanical behavior of the bone, three different mechanical simulation setups were evaluated 1) a cube study, to test the anisotropic information prediction influence on cubic samples of trabecular bone 2) proximal femur, to test the method for predicting the overall behavior of the femur 
bone loaded in stance position and 3) for patella, to study the accuracy of the method in a bone with a complex distribution of trabecular orientations. In all three cases, the results obtained with the registration approach were compared with models considering isotropic material properties. For all the situations, the mechanical parameters calculated with the predicted bone orientation outperform the mechanical results based on an isotropic material assumption.

Other methods have been proposed to estimate bone anisotropy from clinical CT. Hazrati et al. ${ }^{8}$ used mesh registration for finding the correspondences between different proximal femurs and then assign the anisotropic information of the bone in the dataset having the most similar bone density. Lekadir et al. ${ }^{19}$ used the same technique, but instead of taking the anisotropic information from a reference bone, they used a regression method to predict the anisotropic information tensor based on BV/TV and local deformations. In both studies high-resolution $\mu \mathrm{CT}$ scans were employed to obtain this information, which is not applicable in a clinical situation. In addition, the mesh registration method used in these studies works well for long bones, but might fail to provide valid registration for complex anatomical sites such as vertebrae or mandibles. On the contrary, image registration can be used for complex anatomical shapes ${ }^{13,15,29}$. Furthermore, Bonaretti et al. ${ }^{1}$ showed that image registration establishes a better correspondence between different samples compared to mesh registration, which uses only the surface shape of the bone and discards the information inside the bone. As a result, the correspondence established by the models on the element located inside the bone, where the trabecular bone architecture is important, is sub-obtimal ${ }^{1}$. 
An alternative approach is to extract the bone architecture directly from the clinical CT. Kersh et al. ${ }^{14}$ suggested using GST method to extract anisotropic information from patient's CT scan. Larsson et al. ${ }^{17}$ studied different cube sizes for extracting anisotropic information from CT scan and showed that this information is close to the $\mu$ CT based MIL method, when the degree of transverse isotropy (DTI) value is larger than a specific threshold. However, this threshold is large and only covers few regions of interest. For instance, when bone anisotropy was calculated based on $5 \mathrm{~mm}$ spheres, only $1 \%$ of the bone has DTI values greater than the threshold and when the volume to calculate the bone anisotropy was increased to $15 \mathrm{~mm}, 27 \%$ of the bone has a DTI larger than the threshold. However, a radius of $15 \mathrm{~mm}$ represents a large homogenization, which will certainly affect the mechanical simulations. In their study ${ }^{17}$ the mechanical advantage of assigning anisotropic information to only a few regions of the bone $(1 \%$ of the whole bone) was not studied nor compared with results obtained with an isotropic material model.

In the cube study, the anisotropic information was found to be important when the load was applied in the direction of the trabecular bone structure. This result agrees with the findings of Luisier et al. ${ }^{20}$ who found that orthotropic HRpQCT-based FE model improves the isotropic QCT-based results in stance position, which is not the case for side-fall. We found that an isotropic material properties assumption provides FE results close to the ground-truth, especially in the femur, where the thick cortex was modeled with an isotropic material model. However, even in this situation, the template registration clearly improves the estimation of the ultimate failure load as well as the stresses calculated inside the trabecular structures. When analyzing cubes made of 
trabecular bone, the template registration improved the isotropic material results by $10 \%$. Similar results are also seen in Hazrati et al. ${ }^{8}$, where the "cortical" femoral bone shaft was excluded from the simulations.

In this study, we examined the accuracy of our method on predicting the biomechanical behavior of the bone with the assumption of having very accurate bone volume fraction from patient's CT scans. Note that this information is used only for mechanical simulations and not to predict the anisotropic structure. However, to ensure accurate extraction of the bone volume fraction from low-resolution clinical scans, further investigation is required. This aspect is critical since it has been shown that bone volume fraction determines about $90 \%$ of is the most important determinant of the bone elastic properties ${ }^{21}$.

The template bone has been chosen based on the visual inspection of the available datasets in order to select an average healthy bone. To assess the effect of the template selection, the accuracy of the mechanical simulations has been evaluated with respect to the difference between the chosen template and the patient's bone. This evaluation showed that the error of the mechanical prediction remains constant, even when the anatomical difference between the target femur and the template increases (both in term of shape and bone density). This observation indicates that the selection of the bone template does not significantly affect the mechanical prediction regardless of its difference in shape and bone volume fraction. Together with the overall quality of the mechanical simulation, this indicates that the main trabecular orientations remain consistent across different patients. The template bone has been chosen based on visual inspection of the datasets available to select an average non-pathological bone. 
To assess the effect of the template selection, the quality of the mechanical simulation has been evaluated with respect to the difference between the chosen template and the patient's bone. This evaluation showed that the error on the mechanical prediction using the registration approach was constant and was not affected by the difference in the bone shape or bone volume fraction. This observation indicates that the selection of the bone template does not significantly affect the mechanical prediction regardless of its difference in shape and bone volume fraction relative to the patient's bone. Together with the overall quality of the mechanical simulation, this indicates that the main trabecular orientations remain constant across patients. However, this approach remains limited to situations where the bone anisotropy is not affected by specific conditions or diseases or altered by prolonged presence of orthopedic implants. In these cases, alternative template bones corresponding to the specific configuration should be employed.

In the biomechanical simulations we did not consider the anisotropy of the solid portion of the bone ${ }^{11}$ and focused on the anisotropy originating from the micro-structure of the trabecular bone. The orientation and degree of anisotropy of the bone matrix cannot be retrieved from HR-pQCT images but may introduce further differences in the computed biomechanical response. A similar approach could be applied to cortical anisotropy as for trabecular anisotropy, but would require even higher resolution data that are currently not available. In first approximation, we believe that the contribution of the anisotropy of the cortical and trabecular compartments can be investigated independently.

The accuracy of the proposed method has been evaluated using homogenized FE 
(hFE) models. Although this approach represents an approximation of the bone mechanics that requires experimental validation, it does not affect the outcomes of the present numerical technique to determine bone anisotropy.

The proposed method to use image registration to map the bone anisotropy from a template image to the patient clinical dataset improved the mechanical predictions using homogenized FE models. This approach can be used to improve patient-specific bone mechanical simulations based on clinical datasets. As part of future work, the anisotropic information could be extracted from the original template bone and then the fabric tensor could be transformed using the deformation fields calculated from image registration to accelerate the anisotropic information estimation. In addition, to improve the prediction results, the template anisotropy could be calculated as a mean fabric from a large number of bones to further alleviate the possible selection bias produced by the template selection.

\section{Acknowledgment}

The authors would like to thank Dr. Enrico Dall'Ara for preparing, scanning and sharing the femoral data, Dr. Jakob Schwiedrzik for providing the UMAT implementation of the mechanical model, Prof. Dieter Pahr for giving us access to the Medtool software and Dr. Ghislain Maquer and Dr. Hadi Seyed Hosseini for their assistance on the preparation of the FE models.

\section{References}


1. Bonaretti, S., C. Seiler, C. Boichon, M. Reyes, and P. Büchler. Image-based vs. mesh-based statistical appearance models of the human femur: Implications for finite element simulations. Med. Eng. Phys. 36:1626-35, 2014.

2. Boutroy, S., M. L. Bouxsein, F. Munoz, and P. D. Delmas. In vivo assessment of trabecular bone microarchitecture by high-resolution peripheral quantitative computed tomography. J. Clin. Endocrinol. Metab. 90:6508-15, 2005.

3. Burghardt, A. J., T. M. Link, and S. Majumdar. High-resolution computed tomography for clinical imaging of bone microarchitecture. Clin. Orthop. Relat. Res. 469:2179-93, 2011.

4. Doblaré, M., and J. M. García. Application of an anisotropic bone-remodelling model based on a damage-repair theory to the analysis of the proximal femur before and after total hip replacement. J. Biomech. 34:1157-1170, 2001.

5. Dragomir-Daescu, D., J. Op Den Buijs, S. McEligot, Y. Dai, R. C. Entwistle, C. Salas, L. J. Melton, K. E. Bennet, S. Khosla, and S. Amin. Robust QCT/FEA models of proximal femur stiffness and fracture load during a sideways fall on the hip. Ann. Biomed. Eng. 39:742-55, 2011.

6. Enns-Bray, W. S., J. S. Owoc, K. K. Nishiyama, and S. K. Boyd. Mapping 
anisotropy of the proximal femur for enhanced image based finite element analysis. J. Biomech. 47:3272-8, 2014.

7. Harrigan, T. P., and R. W. Mann. Characterization of microstructural anisotropy in orthotropic materials using a second rank tensor. J. Mater. Sci. 19:761-767, 1984.

8. Hazrati Marangalou, J., K. Ito, M. Cataldi, F. Taddei, and B. van Rietbergen. A novel approach to estimate trabecular bone anisotropy using a database approach. J. Biomech. 46:2356-62, 2013.

9. Hazrati Marangalou, J., K. Ito, and B. van Rietbergen. A novel approach to estimate trabecular bone anisotropy from stress tensors. Biomech. Model. Mechanobiol. , 2014.doi:10.1007/s10237-014-0584-6

10. Hellmich, C., C. Kober, and B. Erdmann. Micromechanics-based conversion of CT data into anisotropic elasticity tensors, applied to FE simulations of a mandible. Ann. Biomed. Eng. 36:108-22, 2008.

11. Hellmich, C., F.-J. Ulm, and L. Dormieux. Can the diverse elastic properties of trabecular and cortical bone be attributed to only a few tissue-independent phase properties and their interactions? Arguments from a multiscale approach. 
Biomech. Model. Mechanobiol. 2:219-38, 2004.

12. Horn, B. K. P. Closed-form solution of absolute orientation using unit quaternions. J. Opt. Soc. Am. A 4:629, 1987.

13. Ino, F., Y. Kawasaki, T. Tashiro, Y. Nakajima, Y. Sato, S. Tamura, and K. Hagihara. A Parallel Implementation of 2-D/3-D Image Registration for ComputerAssisted Surgery. , 2005.

14. Kersh, M. E., P. K. Zysset, D. H. Pahr, U. Wolfram, D. Larsson, and M. G. Pandy. Measurement of structural anisotropy in femoral trabecular bone using clinicalresolution CT images. J. Biomech. 46:2659-66, 2013.

15. Klein, S., M. Staring, K. Murphy, M. a Viergever, and J. P. W. Pluim. Elastix: a Toolbox for Intensity-Based Medical Image Registration. IEEE Trans. Med. Imaging 29:196-205, 2010.

16. Kober, C., B. Erdmann, C. Hellmich, R. Sader, and H.-F. Zeilhofer. Consideration of anisotropic elasticity minimizes volumetric rather than shear deformation in human mandible. Comput. Methods Biomech. Biomed. Engin. 9:91-101, 2006.

17. Larsson, D., B. Luisier, M. E. Kersh, E. Dall'ara, P. K. Zysset, M. G. Pandy, and 
D. H. Pahr. Assessment of transverse isotropy in clinical-level CT images of trabecular bone using the gradient structure tensor. Ann. Biomed. Eng. 42:950-9, 2014.

18. Latypova, A., F. Levrero, D. Pioletti, B. Jolles, and A. Terrier. A musculoskeletal numerical knee model to assess patellar resurfacing in total knee arthroplasty. , 2013.at <http://www.ors.org/Transactions/59/PS2--099/1709.html>

19. Lekadir, K., J. Hazrati-Marangalou, C. Hoogendoorn, Z. Taylor, B. van Rietbergen, and A. F. Frangi. Statistical estimation of femur micro-architecture using optimal shape and density predictors. J. Biomech. 48:598-603, 2015.

20. Luisier, B., E. Dall'Ara, and D. H. Pahr. Orthotropic HR-pQCT-based FE models improve strength predictions for stance but not for side-way fall loading compared to isotropic QCT-based FE models of human femurs. J. Mech. Behav. Biomed. Mater. 32:287-99, 2014.

21. Maquer, G., S. N. Musy, J. Wandel, T. Gross, and P. K. Zysset. Bone volume fraction and fabric anisotropy are better determinants of trabecular bone stiffness than other morphological variables. J. Bone Miner. Res. 30:1000-8, 2015.

22. Minh, H. Le, W. M. Park, K. Kim, S.-W. Son, S.-H. Lee, and Y. H. Kim. A new 
patient-specific planning method based on joint contact force balance with soft tissue release in total knee arthroplasty. Int. J. Precis. Eng. Manuf. 14:2193-2199, 2013.

23. Muller, R., T. Hildebrand, and P. Ruegsegger. Non-invasive bone biopsy: a new method to analyse and display the three-dimensional structure of trabecular bone. Phys. Med. Biol. 39:145-164, 1994.

24. Ourselin, S., A. Roche, S. Prima, and N. Ayache. Block Matching: A General Framework to Improve Robustness of Rigid Registration of Medical Images. In: Medical Image Computing and Computer-Assisted Intervention MICCAI 2000 SE - 57, edited by S. Delp, A. DiGoia, and B. Jaramaz. Springer Berlin Heidelberg, 2000, pp. 557-566.

25. Pahr, D. H., and P. K. Zysset. From high-resolution CT data to finite element models: development of an integrated modular framework. Comput. Methods Biomech. Biomed. Engin. 12:45-57, 2009.

26. Rueckert, D., L. I. Sonoda, C. Hayes, D. L. Hill, M. O. Leach, and D. J. Hawkes. Nonrigid registration using free-form deformations: application to breast MR images. IEEE Trans. Med. Imaging 18:712-21, 1999. 
27. San Antonio, T., M. Ciaccia, C. Müller-Karger, and E. Casanova. Orientation of orthotropic material properties in a femur FE model: A method based on the principal stresses directions. Med. Eng. Phys. 34:914-919, 2012.

28. Schwiedrzik, J. J., and P. K. Zysset. An anisotropic elastic-viscoplastic damage model for bone tissue. Biomech. Model. Mechanobiol. 12:201-13, 2013.

29. Seiler, C., X. Pennec, and M. Reyes. Capturing the multiscale anatomical shape variability with polyaffine transformation trees. Med. Image Anal. 16:1371-84, 2012.

30. Tabor, Z., and E. Rokita. Quantifying anisotropy of trabecular bone from graylevel images. Bone 40:966-72, 2007.

31. Takahashi, A., H. Sano, M. Ohnuma, M. Kashiwaba, D. Chiba, M. Kamimura, T. Sugita, and E. Itoi. Patellar morphology and femoral component geometry influence patellofemoral contact stress in total knee arthroplasty without patellar resurfacing. Knee Surg. Sports Traumatol. Arthrosc. 20:1787-95, 2012.

32. The CGAL Project. CGAL User and Reference Manual. CGAL Editorial Board, 2015.at <http://doc.cgal.org/4.6/Manual/packages.html> 
33. Trabelsi, N., and Z. Yosibash. Patient-specific finite-element analyses of the proximal femur with orthotropic material properties validated by experiments. J. Biomech. Eng. 133:061001, 2011.

34. Wolfram, U., B. Schmitz, F. Heuer, M. Reinehr, and H.-J. Wilke. Vertebral trabecular main direction can be determined from clinical CT datasets using the gradient structure tensor and not the inertia tensor--a case study. J. Biomech. 42:1390-6, 2009.

35. Zysset, P. K., and A. Curnier. An alternative model for anisotropic elasticity based on fabric tensors. Mech. Mater. 21:243-250, 1995. 
Table 1: Mechanical parameters for the elastic-visco plastic model used in the finite element simulations.

Table 2. Mechanical results of trabecular cubes, the accuracy has been calculated by comparing the results of the different methods to simulation performed with the actual bone anisotropy. The accuracy of our method on predicting von Mises stress value was compared to the assumption of isotropic material. Simulation results showed that the accuracy of isotropic material drops when the loading is aligned with the main trabecular direction, while simulation performed with using the template registration were not affect by the loading direction. 
Table 1: Mechanical parameters for the elastic-visco plastic model used in the finite element simulations.

\begin{tabular}{|c|c|c|c|c|c|c|c|c|c|c|c|c|}
\hline & & $\begin{array}{c}E_{0} \\
{[\mathrm{GPa}]}\end{array}$ & $v_{0}$ & $\begin{array}{c}G_{0} \\
{[\mathrm{GPa}]}\end{array}$ & $k$ & $l$ & $\begin{array}{c}\sigma_{0} \\
{[\mathrm{MPa}]}\end{array}$ & $\chi_{0}$ & $\begin{array}{c}\tau_{0} \\
{[\mathrm{MPa}]}\end{array}$ & $\eta$ & $\mathrm{m}$ & $\begin{array}{l}\text { Yield } \\
\text { ratio }\end{array}$ \\
\hline Trabecular & Tension & 12 & 0.249 & 3.913 & 1.878 & 1.076 & 81.6 & -0.3 & 68.9 & \multirow{4}{*}{\multicolumn{2}{|c|}{1.24 .0}} & \multirow{4}{*}{0.66} \\
\hline Tranecurat & Compression & & & & & & 111.6 & 0.31 & & & & \\
\hline \multirow[t]{2}{*}{ Cortical } & Tension & 12 & 0.34 & 4.47 & 1.0 & 1.0 & 72.0 & -0.37 & 62.6 & & & \\
\hline & Compres & & & & & & 108.0 & 0.49 & & & & \\
\hline
\end{tabular}


Table 2. Mechanical results of trabecular cubes, the accuracy has been calculated by comparing the results of the different methods to simulation performed with the actual bone anisotropy. The accuracy of our method on predicting von Mises stress value was compared to the assumption of isotropic material. Simulation results showed that the accuracy of isotropic material drops when the loading is aligned with the main trabecular direction, while simulation performed with using the template registration were not affect by the loading direction.

\begin{tabular}{|c|c|c|c|c|}
\hline \multirow{2}{*}{ Femur No. } & \multicolumn{2}{|c|}{$\begin{array}{c}\text { Average accuracy for all loading } \\
\text { conditions }(\%)\end{array}$} & \multicolumn{2}{|c|}{$\begin{array}{l}\text { Accuracy for loadings in the main } \\
\text { trabecular direction only }(\%)\end{array}$} \\
\hline & $\begin{array}{c}\text { Template } \\
\text { Registration } \\
\end{array}$ & Isotropic & $\begin{array}{c}\text { Template } \\
\text { Registration } \\
\end{array}$ & Isotropic \\
\hline 1. & 10.0 & 13.3 & 8.6 & 19.3 \\
\hline 2. & 10.7 & 15.7 & 9.9 & 20.1 \\
\hline 3. & 8.1 & 13.0 & 7.1 & 18.0 \\
\hline 4. & 10.3 & 14.1 & 10.7 & 21.5 \\
\hline 5. & 7.5 & 13.7 & 7.4 & 17.1 \\
\hline 6. & 7.6 & 13.7 & 6.7 & 18.3 \\
\hline 7. & 9.1 & 13.4 & 7.0 & 18.9 \\
\hline 8. & 11.7 & 13.7 & 16.7 & 18.1 \\
\hline 9. & 7.9 & 14.1 & 8.0 & 18.3 \\
\hline 10. & 8.7 & 13.4 & 7.8 & 17.0 \\
\hline Median & 8.9 & $\overline{c 13.7}$ & $\overline{c 7.9}$ & 18.3 \\
\hline Average & 9.2 & 13.8 & 9.0 & 18.7 \\
\hline STD & 1.4 & 0.7 & 3.0 & 1.4 \\
\hline $\mathrm{p}$-value (t-test) & & $2.0 \mathrm{E}-6$ & & $3.2 \mathrm{E}-6$ \\
\hline
\end{tabular}


Figure 1. Schematic representation of the image registration pipeline. One healthy bone is selected as template. The HRpQCT and QCT scan of the template bone are acquired. First, the QCT scan is registered to the patient's low-resolution CT scan, then obtained deformation fields are applied to the HRpQCT image. The resulting image is then used to estimate bone anisotropic for this patient.

Figure 2. Pipeline describing the study on isolated bones of trabecular bone. Cubic samples were cropped in the center of the femoral head. The anisotropic information was extracted and assigned to each element of the cube mesh. Three compression and three shear loadings were applied. 36

Figure 3. Description of the simulation performed on the femur. Each bone was meshed using an automatic procedure. A grid-based algorithm was then used to extract anisotropic information of the trabecular bone and assign the local bone fabric to each finite element of the mesh. The elastic-visco plastic material model, which relies on the bone anisotropy, was used to calculate material properties of the bone loaded in stance position.

Figure 4. The simulations performed on the patella relied on the non-rigid registration of the template $\mu \mathrm{CT}$ to the trabecular bone of the patient. Forces applied on the patellar tendon and quadriceps corresponded to a $60^{\circ}$ position of a squat movement. 38

Figure 5. Typical results of high-resolution images registered to the shape of the patient's bone for the femur (b) and patella (b). The result of image registration is overlapped with the ground-truth high-resolution scans. As it can be seen around 
the intersecting line, the fiber directions of the registered image follow the directions of the bone trabeculae of the ground-truth image.

Figure 6. Force-displacement curve (left) and contour plot of the failure criterion (right) obtained on one femur. The template registration was better at simulating the mechanical effect than the isotropic model, which underestimated the failure load.

Figure 7. For each femur, the ultimate force and displacement values were calculated and compared to the ground-truth calculations. Values obtained with using the template registration showed a high correspondence to the ultimate force calculated directly from the high-resolution images with a slope of 0.99 . The isotropic model consistently underestimated the ultimate load (slope of 0.94 ) and ultimate displacement (slope 0.93). 41

Figure 8. Principal logarithmic strain in the patella obtained with the different models. The correlation between the ground-truth, the template registration and isotropic model is shown on the left, while the contour plot on the right shows the strain distribution in the patella models. Results showed that the isotropic model overestimates the bone strain, while the template registration improved the strain predictions. ANCOVA test showed that the slope difference for isotropic material result and the results of our method are statically significant with p-value smaller than 0.001 . 


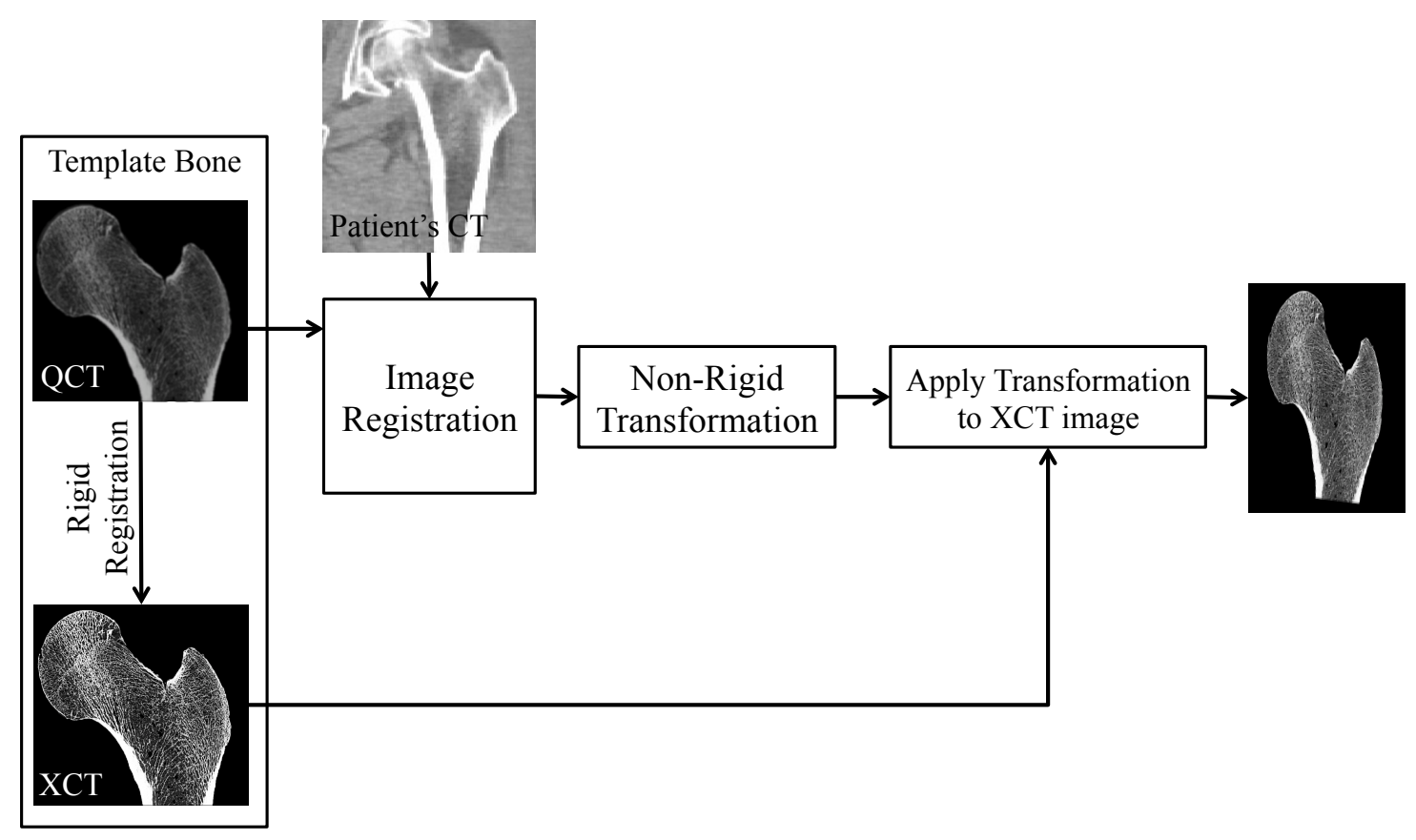

Figure 1. Taghizadeh, Elham 

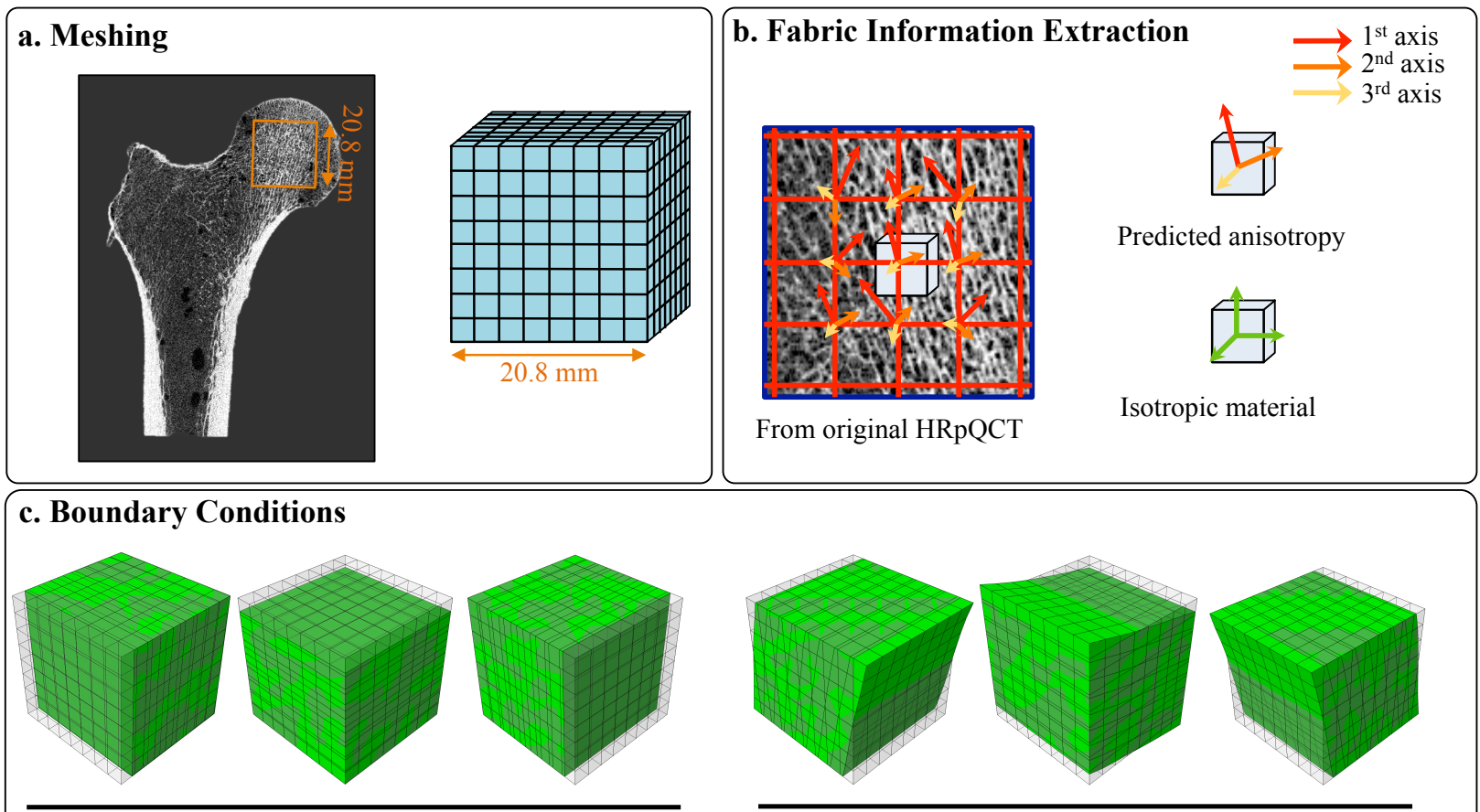

Compression
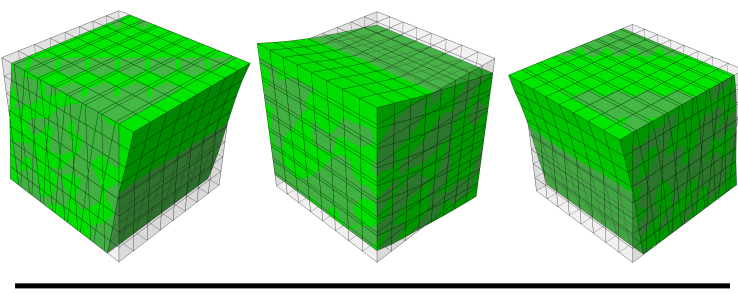

Shear

Figure 2. Taghizadeh, Elham 


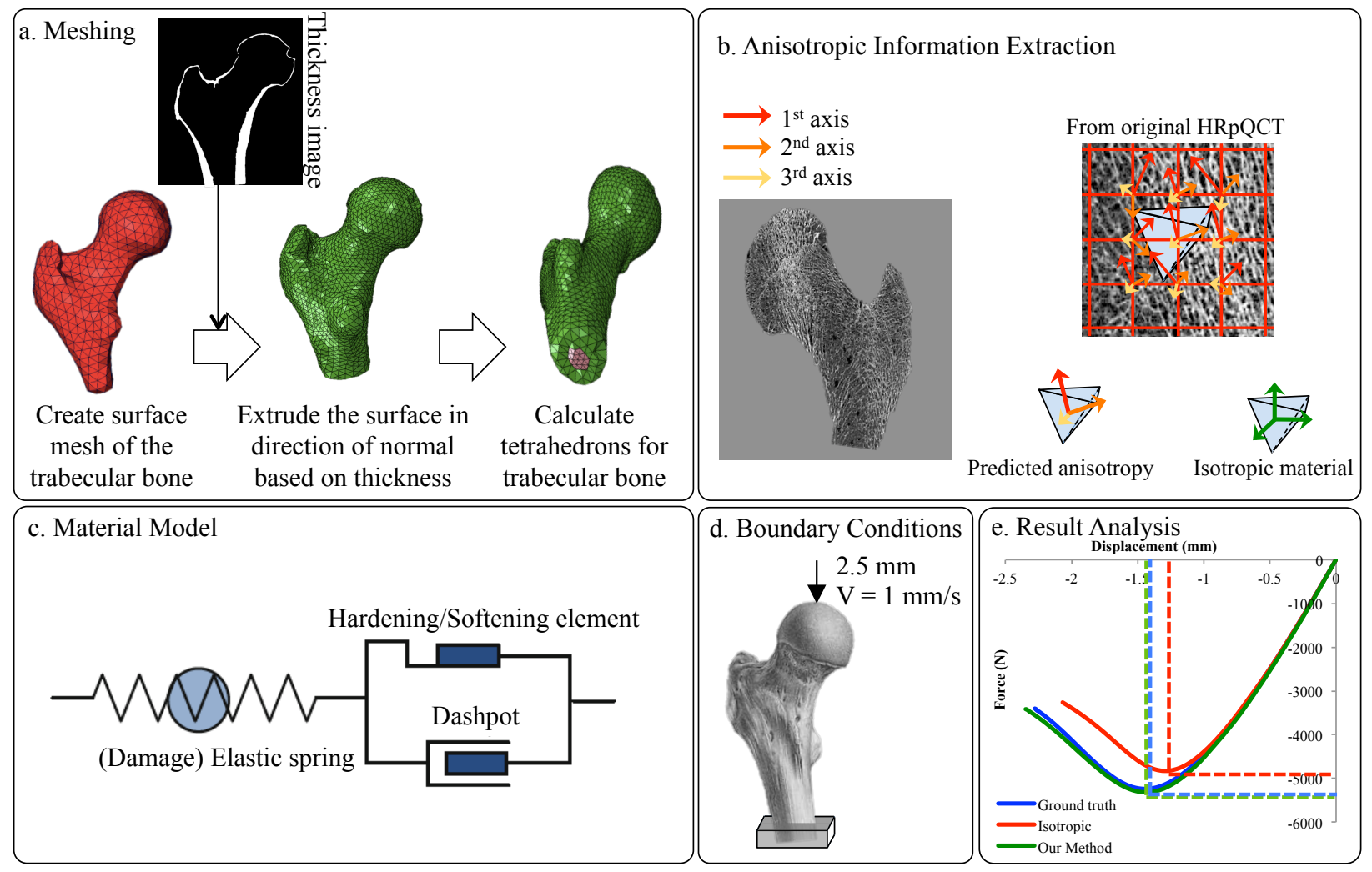

Figure 3. Taghizadeh, Elham 

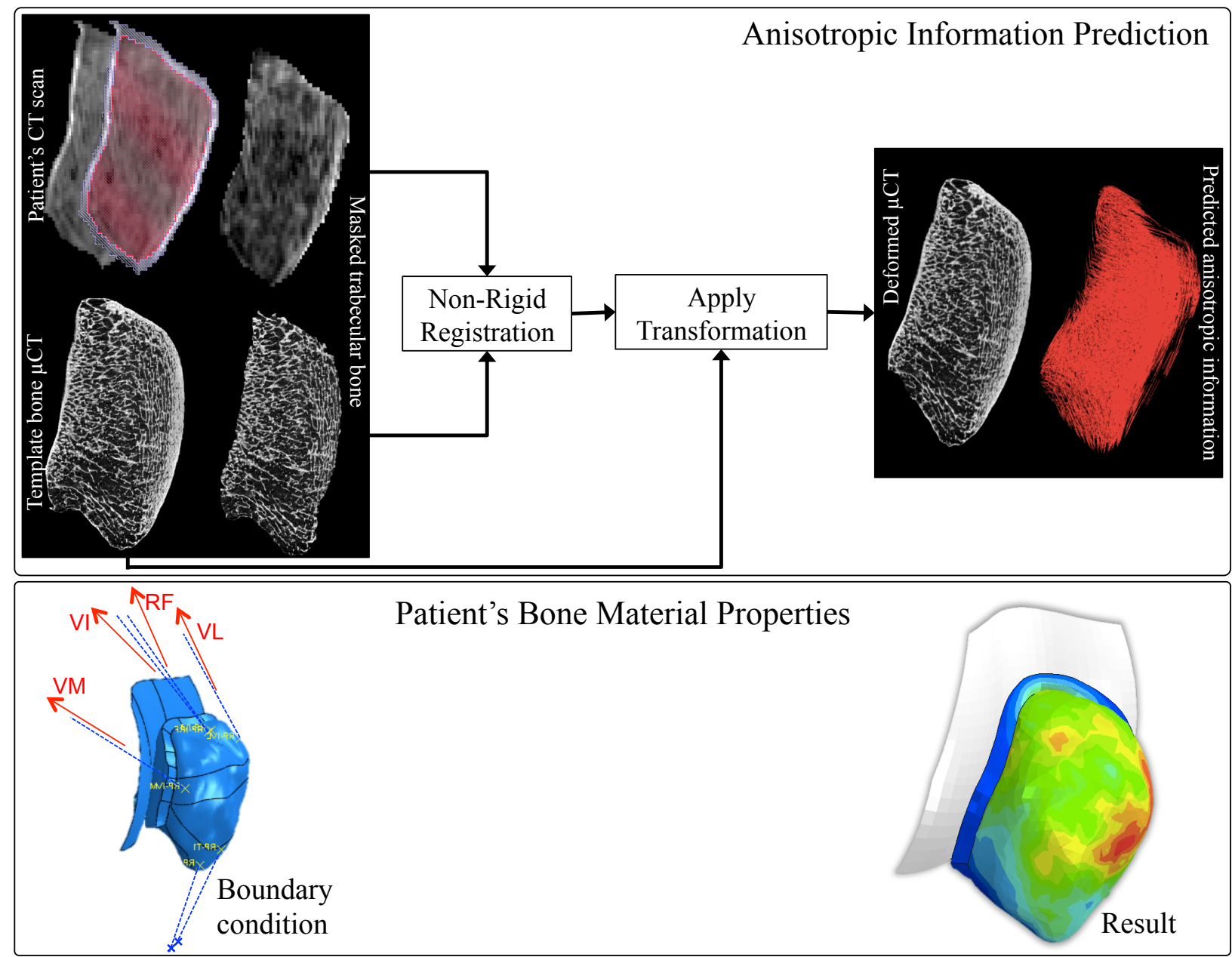

Figure 4. Taghizadeh, Elham 


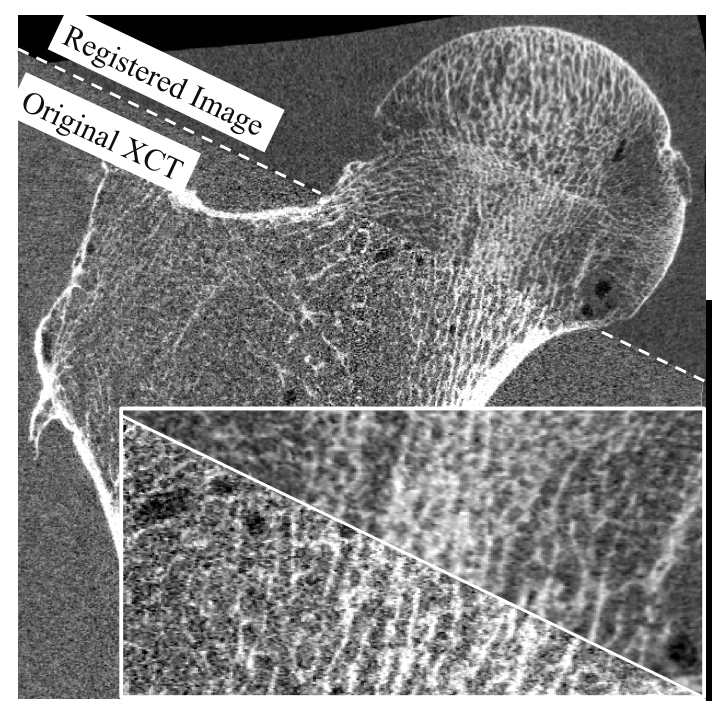

(a)

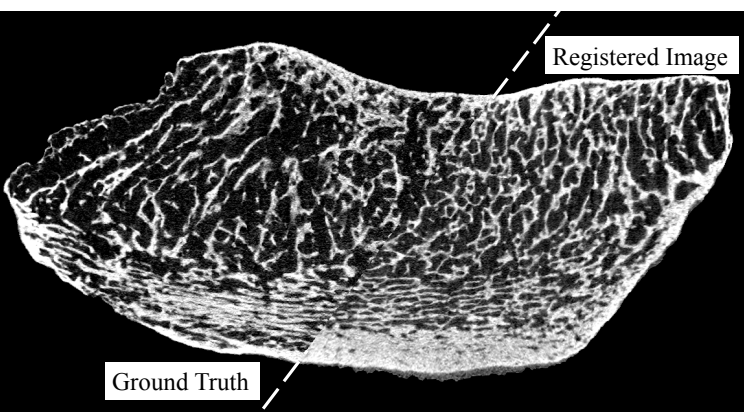

(b)

Figure 5. Taghizadeh, Elham 


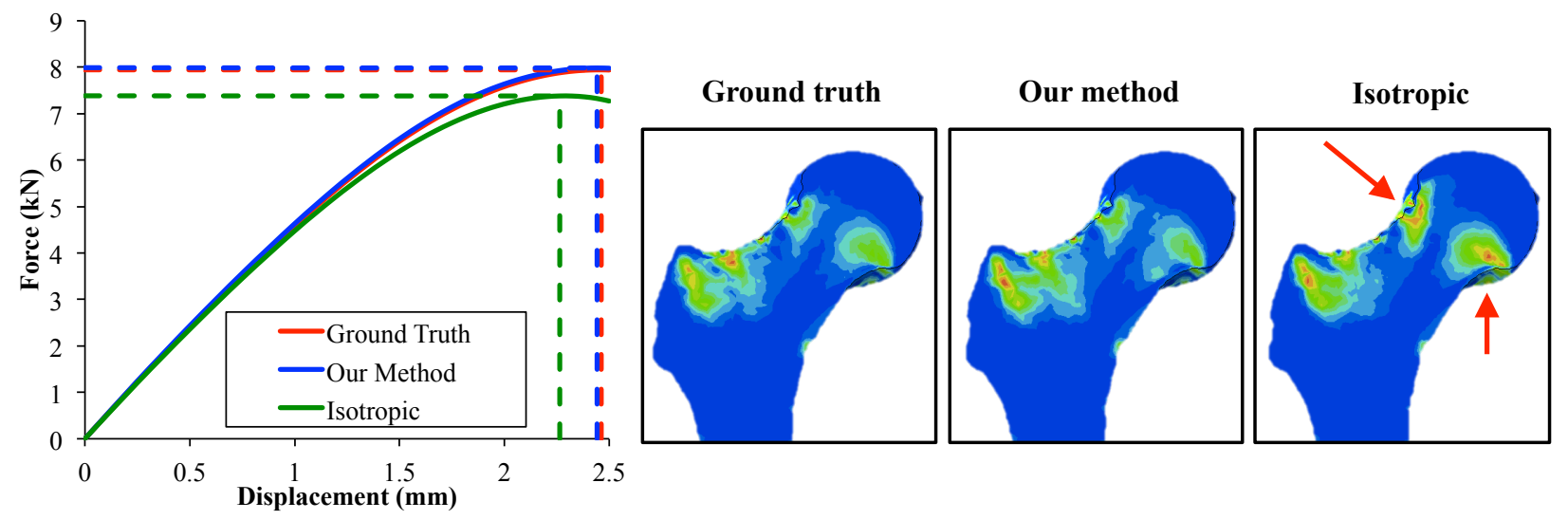

Figure 6. Taghizadeh, Elham 


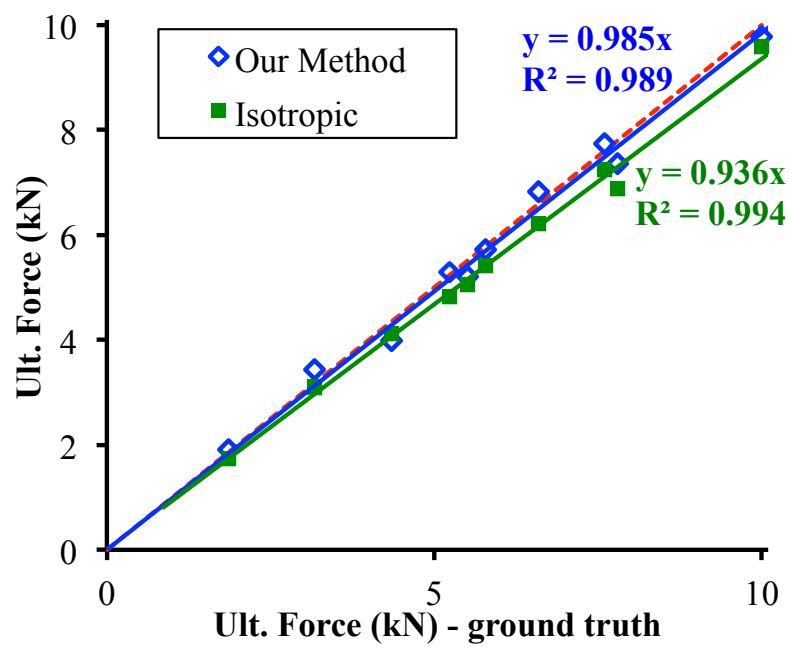

(a)

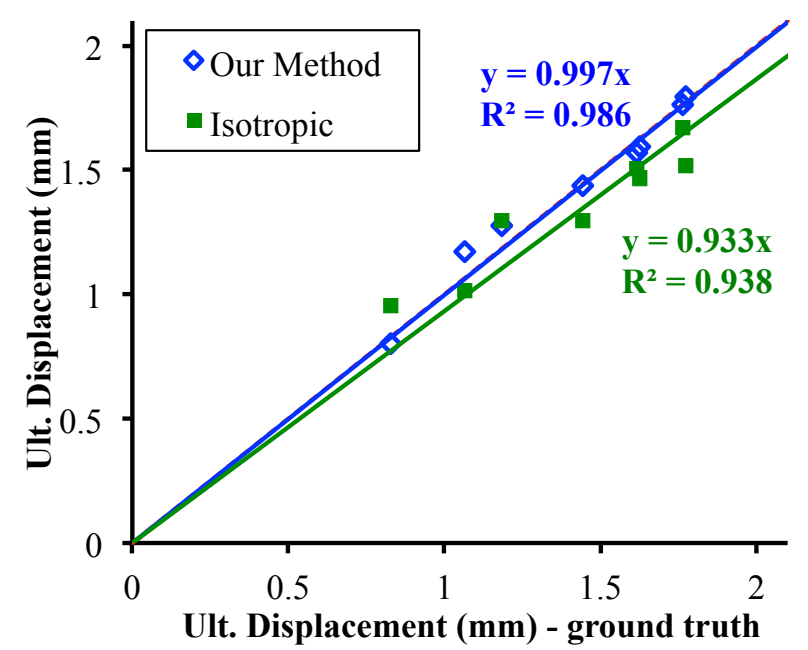

(b)

Figure 7. Taghizadeh, Elham 

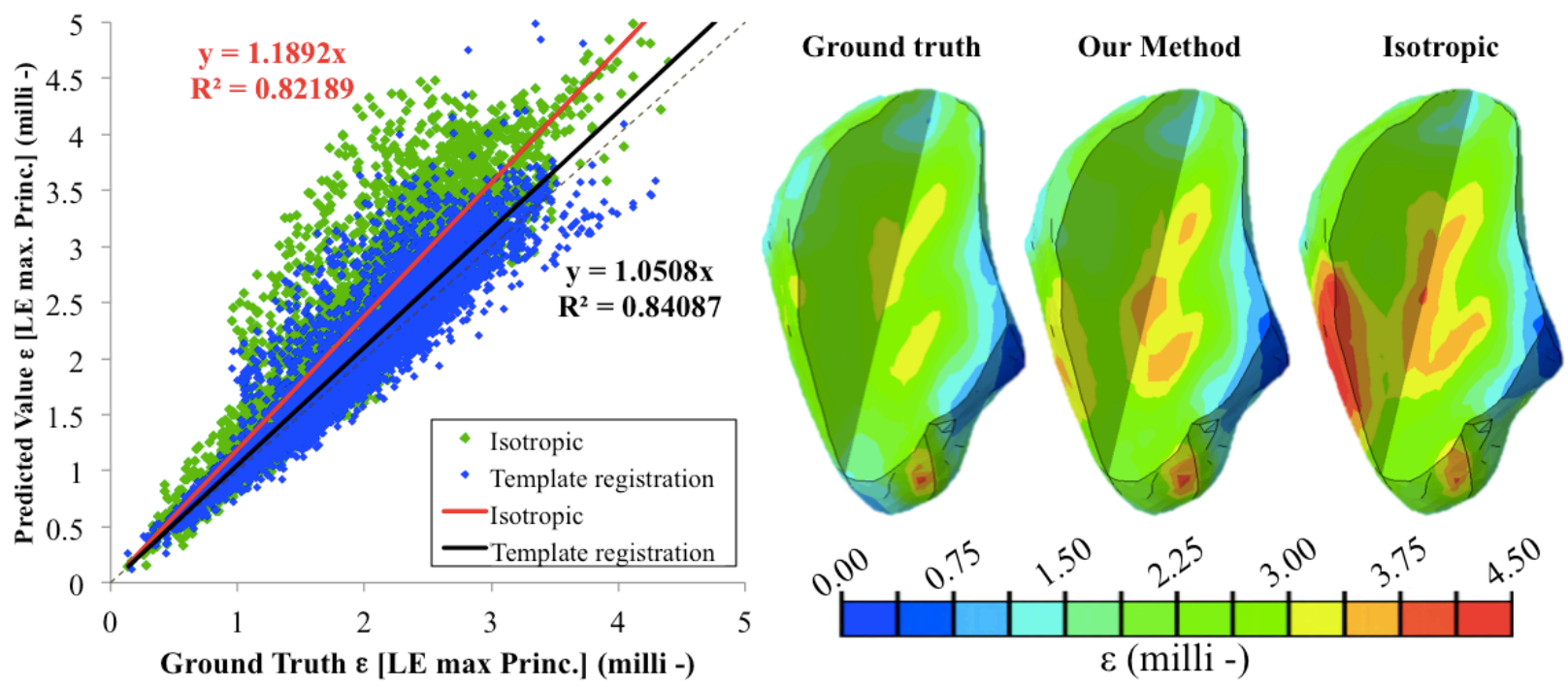

Figure 8. Taghizadeh, Elham 